\title{
Scalar Green's Functions in an Euclidean Space with a Conical-Type Line Singularity
}

\author{
M. E. X. Guimarães ${ }^{\star}$, B. Linet \\ Laboratoire de Gravitation et Cosmologie Relativistes, CNRS/URA 769, \\ Université Pierre et Marie Curie, Tour 22/12, Boîte Courrier 142, 4, Place Jussieu, \\ F-75252 Paris Cedex 05, France, e-mail: marg@ccr.jussieu.fr
}

Received: 11 October 1993/in revised form: 10 February 1994

\begin{abstract}
In an Euclidean space with a conical-type line singularity, we determine the Green's function for a charged massive scalar field interacting with a magnetic flux running through the line singularity. We give an integral expression of the Green's function and a local form in the neighbourhood of the point source, where it is the sum of the usual Green's function in Euclidean space and a regular term. As an application, we derive the vacuum energy-momentum tensor in the massless case for an arbitrary magnetic flux.
\end{abstract}

\section{Introduction}

We consider an Euclidean space with a conical-type line singularity which is described by the metric

$$
d s^{2}=\left(d x^{1}\right)^{2}+\cdots+\left(d x^{n-2}\right)^{2}+d \rho^{2}+B^{2} \rho^{2} d \varphi^{2}
$$

in a coordinate system $\left(x^{i}, \rho, \varphi\right), i=1, \ldots, n-2$, such that $\rho \geq 0$ and $0 \leq \varphi<2 \pi$, the hypersurface $\varphi=0$ and $\varphi=2 \pi$ being identified. Metric (1.1) is characterized by an arbitrary constant $B$, different from zero, and it is globally Euclidean for $B=1$.

Riemannian metric (1.1) may result from the complexification of the time coordinate of a spacetime by a Wick rotation. In the Einstein theory of gravitation, this spacetime having a conical-type line source represents in three dimensions a point mass [1] and in four dimensions a straight cosmic string [2]; the constant $B$ being determined by

$$
B=1-\frac{4 G}{c^{2}} \mu
$$

where $\mu$ is either the mass of the point mass or the linear mass density of the cosmic string.

* Supported by a grant from CNPq (Brazilian government agency FA) 
Presently, attention has been focused on the physical aspects of studying a charged scalar field in the presence of a cosmic string which carries an internal magnetic flux; once this situation reproduces an Aharonov-Bohm effect generalization. We thus consider a charged scalar field $\Psi\left(x^{i}, \rho, \varphi\right)$, with charge $e$, interacting with a magnetic flux $\Phi$ running through the axis $\rho=0$ of metric (1.1). In the usual manner [3, 4], the electromagnetic component $A_{\varphi}$, giving this magnetic flux, can be eliminated outside the axis $\rho=0$ by a gauge transformation

$$
\Psi^{\prime}\left(x^{i}, \rho, \varphi\right)=e^{\imath \varphi \Phi / \Phi_{0}} \Psi\left(x^{i}, \rho, \varphi\right),
$$

where $\Phi_{0}$ is the quantum flux $2 \pi \hbar c / e$. Then, the new scalar field $\Psi^{\prime}\left(x^{2}, \rho, \varphi\right)$ obeys the covariant Laplace equation and satisfies the following requirements:

$$
\begin{aligned}
\Psi^{\prime}\left(x^{i}, \rho, \varphi+2 \pi\right) & =e^{2 i \pi \Phi / \Phi_{0}} \Psi^{\prime}\left(x^{i}, \rho, \varphi\right), \\
\left(\frac{\partial}{\partial \varphi} \Psi^{\prime}\right)\left(x^{i}, \rho, \varphi+2 \pi\right) & =e^{2 \imath \pi \Phi / \Phi_{0}}\left(\frac{\partial}{\partial \varphi} \Psi^{\prime}\right)\left(x^{2}, \rho, \varphi\right),
\end{aligned}
$$

which permit to determine the solution $\Psi^{\prime}\left(x^{\imath}, \rho, \varphi\right)$ to the Laplace equation.

The aim of this paper is to determine the Green's function $G_{\Phi}^{(n)}\left(x, x_{0} ; m\right)$ for the covariant Laplace equation in the space described by metric (1.1)

$$
\begin{aligned}
& {\left[\frac{\partial^{2}}{\partial\left(x^{1}\right)^{2}}+\cdots+\frac{\partial^{2}}{\partial\left(x^{n-2}\right)^{2}}+\frac{\partial^{2}}{\partial \rho^{2}}+\frac{1}{\rho} \frac{\partial}{\partial \rho}+\frac{1}{B^{2} \rho^{2}} \frac{\partial^{2}}{\partial \varphi^{2}}-m^{2}\right] G_{\Phi}^{(n)}} \\
& \quad=-\frac{1}{B} \delta^{(n)}\left(x, x_{0}\right)
\end{aligned}
$$

subject to requirements (1.3), the conical-type line singularity carrying flux $\Phi$. We impose that $G_{\Phi}^{(n)}\left(x, x_{0} ; m\right)$ vanishes when the points $x$ and $x_{0}$ are infinitely separated. We do not touch the question of whether boundary conditions need to be imposed at the conical singularity $\rho=0[5,6]$. In the case where the mass $m$ vanishes, Dowker $[7,8]$ has already written this type of Green's function in metric (1.1) as contour integrals in the complex plane. Our main contribution is to treat the massive case.

We will give an integral expression of the $G_{\Phi}^{(n)}\left(x, x_{0} ; m\right)$ and also a local form in the neighbourhood of the point source in which $G_{\Phi}^{(n)}\left(x, x_{0} ; m\right)$ is the sum of the usual Green's function and a regular term. We anticipate and we mention that this local form is valid when the points $x$ and $x_{0}$ belong to the subset of the space defined by

$$
\frac{\pi}{B}-2 \pi<\varphi-\varphi_{0}<2 \pi-\frac{\pi}{B}
$$

in the case $B>1 / 2$ in which we restrict ourselves.

Metric (1.1) is locally flat but its geometry is non-trivial [9]. So, some interesting quantum effects may occur such as vacuum polarization of the energy-momentum tensor [10-12], within the quantum field theory in curved spacetime [13]. There is the advantage of working in the Euclidean approach [14], where in the free case the fundamental quantity is the Euclidean Green's function. As an application of our results, we evaluate the vacuum expectation values of the energy-momentum tensor in four dimensions either for the massless or the massive scalar field. This calculus is straightforward and consists in taking the coincidence limit $x=x_{0}$ of the regular term and its derivatives in the local form of the Euclidean Green's function.

The plan of the work is as follows. In Sect. 2, we give the recurrence relation between the Green's functions. In Sect. 3, we determine the integral expression of 
$G_{\Phi}^{(2)}\left(x, x_{0} ; m\right)$. We can then derive in Sect. 4 the integral expression of $G_{\Phi}^{(n)}\left(x, x_{0} ; m\right)$. In Sect. 5, we find a local expression of $G_{\Phi}^{(2)}\left(x, x_{0} ; m\right)$. We deduce in Sect. 6 a local expression of $G_{\Phi}^{(n)}\left(x, x_{0} ; m\right)$. In Sect. 7 , as an application for $n=4$, we obtain the asymptotic form of the vacuum energy- momentum tensor at large distance; in the massless case, we give the explicit expression of this tensor. We add in Sect. 8 some concluding remarks.

\section{Preliminaries}

Metric (1.1) takes the Euclidean form in cylindical coordinates when we perform the change of coordinate

$$
\theta=B \varphi
$$

We can determine the Green's function in the subset of the Euclidean space covered by the coordinate system $\left(x^{2}, \rho, \theta\right)$ with $0 \leq \theta<2 \pi B$. Equation (1.4) becomes the usual Laplace equation

$$
\left(\triangle-m^{2}\right) \tilde{G}_{\Phi}^{(n)}=-\delta^{(n)}\left(x, x_{0}\right)
$$

where $\tilde{G}_{\Phi}^{(n)}$ is defined by

$$
\tilde{G}_{\Phi}^{(n)}\left(x^{2}, \rho, \theta\right)=G_{\Phi}^{(n)}\left(x^{i}, \rho, \theta / B\right)
$$

for $0 \leq \theta<2 \pi B$. In order to ensure requirements (1.3), we impose the following boundary conditions on the hypersurfaces $\theta=0$ and $\theta=2 \pi B$,

$$
\begin{aligned}
\tilde{G}_{\Phi}^{(n)}\left(x^{i}, \rho, 2 \pi B\right) & =e^{2 i \pi \Phi / \Phi_{0}} \tilde{G}_{\Phi}^{(n)}\left(x^{i}, \rho, 0\right), \\
\left(\frac{\partial}{\partial \theta} \tilde{G}_{\Phi}^{(n)}\right)\left(x^{\imath}, \rho, 2 \pi B\right) & =e^{2 \imath \pi \Phi / \Phi_{0}}\left(\frac{\partial}{\partial \theta} \tilde{G}_{\Phi}^{(n)}\right)\left(x^{i}, \rho, 0\right) .
\end{aligned}
$$

It is to be remarked that we can only consider a positive flux $\Phi$ since the negative case is obtained by taking the complex conjugate. Futhermore, only the fractional part of $\Phi / \Phi_{0}$, denoted $\gamma$, occurs in boundary conditions (2.4). Therefore, the Green's function is noted $\tilde{G}_{\gamma}^{(n)}$ for $0 \leq \gamma<1$. The case $\gamma=0$ corresponds to the ordinary Green's function. The case $\gamma=\frac{1}{2}$ describes a twisted scalar field around the axis $\rho=0$, [11] and references therein.

The important thing to notice is that a recurrence relation between the Green's functions can be easily proved

$$
\begin{aligned}
& \tilde{G}_{\gamma}^{(n)}\left(x^{1}, \ldots, x^{n-2}, \rho, \theta ; m\right) \\
& \quad=\frac{1}{2 \pi} \int_{-\infty}^{\infty} \tilde{G}_{\gamma}^{(n-1)}\left(x^{1}, \ldots, x^{n-3}, \rho, \theta ; \sqrt{m^{2}+\lambda^{2}}\right) \cos \left[\lambda\left(x^{n-2}-x_{0}^{n-2}\right)\right] d \lambda
\end{aligned}
$$

for $n \geq 3$, by making use of the identity

$$
\delta\left(x^{n-2}-x_{0}^{n-2}\right)=\frac{1}{2 \pi} \int_{-\infty}^{\infty} \cos \left[\lambda\left(x^{n-2}-x_{0}^{n-2}\right)\right] d \lambda .
$$


Recurrence relation (2.5) conserves boundary conditions (2.4). In consequence, the first problem is to find $\tilde{G}_{\gamma}^{(2)}$. After this determination, we return to coordinate $\varphi$ to get the Green's function $G_{\gamma}^{(2)}\left(x, x_{0} ; m\right)$ and then we can determine all the Green's functions $G_{\gamma}^{(n)}\left(x, x_{0} ; m\right)$.

\section{Integral Expression of $G_{\gamma}^{(2)}\left(x, x_{0} ; m\right)$}

In the Euclidean space covered by coordinates $(\rho, \theta)$ with $0 \leq \theta<2 \pi B$, the Green's function $\tilde{G}_{\gamma}^{(2)}$ obeys Eq. (2.2) which reduces to

$$
\left(\frac{\partial^{2}}{\partial \rho^{2}}+\frac{1}{\rho} \frac{\partial}{\partial \rho}+\frac{1}{\rho^{2}} \frac{\partial^{2}}{\partial \theta^{2}}-m^{2}\right) \tilde{G}_{\gamma}^{(2)}=-\delta^{(2)}\left(\rho, \theta, \rho_{0}, \theta_{0}\right)
$$

and it must verify boundary conditions (2.4).

The usual Green's function of equation (3.1) is

$$
\frac{1}{2 \pi} K_{0}\left(m r_{2}\right)
$$

where $r_{2}$ is the Euclidean distance between the two points $(\rho, \theta)$ and $\left(\rho_{0}, \theta_{0}\right)$

$$
r_{2}=\sqrt{\rho^{2}+\rho_{0}^{2}-2 \rho \rho_{0} \cos \left(\theta-\theta_{0}\right)}
$$

and $K_{\mu}$ denotes the modified Bessel functions of the second kind. Of course, it does not satisfy boundary conditions (2.4). To do this, we write down the usual Green's function as an integral [15]

$$
K_{0}\left(m r_{2}\right)=\frac{1}{\pi} \int_{-\infty}^{\infty} K_{i \nu}(m \rho) K_{i \nu}\left(m \rho_{0}\right) e^{\pi \nu} e^{-\nu\left|\theta-\theta_{0}\right|} d \nu
$$

Now the set of homogeneous solutions to Eq. (3.1) is formed with functions $K_{i \nu}(m \rho) e^{\nu \theta}$ and $K_{i \nu}(m \rho) e^{-\nu \theta}, \nu$ real, therefore the general solution to Eq. (3.1) can be written

$$
\tilde{G}_{\gamma}^{(2)}=\frac{1}{2 \pi^{2}} \int_{-\infty}^{\infty} K_{i \nu}(m \rho) K_{i \nu}\left(m \rho_{0}\right) e^{\pi \nu} U_{\nu}(\theta) d \nu
$$

where $U_{\nu}(\theta)$ has the form

$$
U_{\nu}(\theta)=e^{-\nu\left|\theta-\theta_{0}\right|}+C_{1}(\nu) e^{\nu \theta}+C_{2}(\nu) e^{-\nu \theta} .
$$

We choose the coefficients $C_{1}(\nu)$ and $C_{2}(\nu)$ in order to satisfy boundary conditions (2.4) which can be written

$$
\begin{aligned}
U_{\nu}(2 \pi B) & =e^{2 i \pi \gamma} U_{\nu}(0) \\
\left(\frac{\partial}{\partial \theta} U_{\nu}\right)(2 \pi B) & =e^{2 \imath \pi \gamma}\left(\frac{\partial}{\partial \theta} U_{\nu}\right)(0)
\end{aligned}
$$


Inserting (3.4) into (3.5), we find easily

$$
\begin{aligned}
& C_{1}(\nu)=\frac{e^{-\nu \theta_{0}-\pi \nu B+i \pi \gamma}}{e^{\pi \nu B-\imath \pi \gamma}-e^{-\pi \nu B+\imath \pi \gamma}}, \\
& C_{2}(\nu)=\frac{e^{\nu \theta_{0}-\pi \nu B-\imath \pi \gamma}}{e^{\pi \nu B+i \pi \gamma}-e^{-\pi \nu B-i \pi \gamma}},
\end{aligned}
$$

and the function $U_{\nu}(\theta)$ has the expression

$$
U_{\nu}(\theta)=\frac{e^{2 i \pi \gamma} \sinh \left(\nu\left|\theta-\theta_{0}\right|\right)-\sinh \left(\nu\left|\theta-\theta_{0}\right|-2 \pi \nu B\right)}{\cosh (2 \pi \nu B)-\cos (2 \pi \gamma)} .
$$

For $\gamma=0$ and $B=1$, expression (3.7) reduces to

$$
U_{\nu}(\theta)=\frac{\cosh \left(\nu\left|\theta-\theta_{0}\right|-\pi \nu\right)}{\sinh (\pi \nu)}
$$

which gives integral expression (3.2) of the usual Green's function. A more practical form of integral (3.3) is obtained by using the fact that $U_{\nu}(\theta)=-U_{-\nu}(\theta)$; we thus have

$$
\tilde{G}_{\gamma}^{(2)}=\frac{1}{\pi^{2}} \int_{-\infty}^{\infty} K_{\imath \nu}(m \rho) K_{i \nu}\left(m \rho_{0}\right) \sinh (\pi \nu) U_{\nu}(\theta) d \nu
$$

Taking into account the asymptotic behaviour of $K_{i \nu}(m \rho)$ for large $\rho$, we see that $\tilde{G}_{\gamma}^{(2)}$ given by integral (3.9) vanishes when $(\rho, \theta)$ and $\left(\rho_{0}, \theta_{0}\right)$ are infinitely separated. The single unique Green's function $\tilde{G}_{\gamma}^{(2)}$ satisfying the prescribed boundary conditions is thereby established.

A more convenient form for $\tilde{G}_{\gamma}^{(2)}$ is obtained by using the following integral expression of the product of two Bessel functions which is proved in the paper of Garnir [16]

$$
\begin{aligned}
& \sinh (\pi \nu) K_{u \nu}(m \rho) K_{i \nu}\left(m \rho_{0}\right) \\
& =\frac{\pi}{2} \int_{\xi_{2}}^{\infty} J_{0}\left[m\left(2 \rho \rho_{0}\right)^{1 / 2}\left(\cosh u-\cosh \xi_{2}\right)^{1 / 2}\right] \sin (\nu u) d u
\end{aligned}
$$

with the positive quantity $\xi_{2}$ defined by

$$
\cosh \xi_{2}=\frac{\rho^{2}+\rho_{0}^{2}}{2 \rho \rho_{0}},
$$

and in which $J_{\mu}$ is the Bessel function. Insertion of this identity into (3.9) gives

$$
\begin{aligned}
\tilde{G}_{\gamma}^{(2)}= & \frac{1}{2 \pi} \int_{\xi_{2}}^{\infty} d u J_{0}\left[m\left(2 \rho \rho_{0}\right)^{1 / 2}\left(\cosh u-\cosh \xi_{2}\right)^{1 / 2}\right] \\
& \times \int_{0}^{\infty} U_{\nu}(\theta) \sin (\nu u) d \nu .
\end{aligned}
$$


The final result is obtained by finding the Fourier sine transform of the function $U_{\nu}(\theta)$ given by (3.7). To this end, we use the formula [15]

$$
\begin{aligned}
& \int_{0}^{\infty} \frac{\sin (a \nu) \sinh (b \nu)}{\cosh (c \nu)+\cos d} d \nu \\
& \quad=\pi \frac{\sin [b(\pi-d) / c] \sinh [a(\pi+d) / c]-\sin [b(\pi+d) / c] \sinh [a(\pi-d) / c]}{c \sin d[\cosh (2 \pi a / c)-\cos (2 \pi b / c)]}
\end{aligned}
$$

with the conditions of validity: $a>0,|b|<c$ and $|d|<\pi$. Also, the present proof excludes the case $\gamma=0$. However the final expression will give the known result in the limit where $\gamma$ goes to zero. For $0<\gamma<1$, we obtain

$$
\begin{aligned}
& \int_{0}^{\infty} U_{\nu}(\theta) \sin (\nu u) d \nu=\frac{1}{2 B\left[\cosh (u / B)-\cos \left(\left(\theta-\theta_{0}\right) / B\right)\right]} \\
& \quad \times\left\{e^{i\left(\theta-\theta_{0}\right) \gamma / B} \sinh [u(1-\gamma) / B]+e^{-\imath\left(\theta-\theta_{0}\right)(1-\gamma) / B} \sinh [u \gamma / B]\right\}
\end{aligned}
$$

We now return to express $G_{\gamma}^{(2)}\left(x, x_{0} ; m\right)$, in the case $m \neq 0$, where the coordinate $\varphi$ is related to $\theta$ by (2.1); it has the integral expression

$$
G_{\gamma}^{(2)}=\frac{1}{4 \pi B} \int_{\xi_{2}}^{\infty} J_{0}\left[m\left(2 \rho \rho_{0}\right)^{1 / 2}\left(\cosh u-\cosh \xi_{2}\right)^{1 / 2}\right] g_{\gamma}^{(2)}\left(u, \varphi-\varphi_{0}\right) d u
$$

where $\xi_{2}$ is given by (3.10) and the function $g_{\gamma}^{(2)}(u, \psi)$ is deduced from (3.12)

$$
g_{\gamma}^{(2)}(u, \psi)=\frac{e^{\imath \psi \gamma} \sinh [u(1-\gamma) / B]+e^{-i \psi(1-\gamma)} \sinh [u \gamma / B]}{\cosh (u / B)-\cos \psi}
$$

Having determined the integral expression (3.13), we check that $G_{\gamma}^{(2)}\left(x, x_{0} ; m\right)$ satisfies requirements (1.3). Setting $\gamma=0$ in (3.13), we find again the ordinary Green's function due to Garnir [16].

\section{Integral Expression of $G_{\gamma}^{(n)}\left(x, x_{0} ; m\right)$}

We are now in a position to determine the $G_{\gamma}^{(n)}\left(x, x_{0} ; m\right), n \geq 3$, with the aid of recurrence relation (2.5).

For the first step $n=3$, taking into account expression (3.13), we have to know the Fourier cosine transform [15]

$$
\begin{aligned}
& \int_{-\infty}^{\infty} J_{0}\left[A\left(m^{2}+\lambda^{2}\right)^{1 / 2}\right] \cos \left[\lambda\left(x^{1}-x_{0}^{1}\right)\right] d \lambda \\
& = \begin{cases}2 \cos \left[m\left(A^{2}-\left(x^{1}-x_{0}^{1}\right)^{2}\right)^{1 / 2}\right] /\left(A^{2}-\left(x^{1}-x_{0}^{1}\right)^{2}\right)^{1 / 2} & A>x^{1}-x_{0}^{1} \\
0 & A<x^{1}-x_{0}^{1}\end{cases}
\end{aligned}
$$


We thus have

$$
\begin{aligned}
G_{\gamma}^{(3)}= & \frac{1}{4 \pi^{2} B\left(2 \rho \rho_{0}\right)^{1 / 2}} \int_{\xi_{3}}^{\infty} \frac{\cos \left[m\left(2 \rho \rho_{0}\right)^{1 / 2}\left(\cosh u-\cosh \xi_{3}\right)^{1 / 2}\right]}{\left(\cosh u-\cosh \xi_{3}\right)^{1 / 2}} \\
& \times g_{\gamma}^{(2)}\left(u, \varphi-\varphi_{0}\right) d u,
\end{aligned}
$$

where the positive quantity $\xi_{3}$ is defined by

$$
\cosh \xi_{3}=\frac{\rho^{2}+\rho_{0}^{2}+\left(x^{1}-x_{0}^{1}\right)^{2}}{2 \rho \rho_{0}},
$$

$g_{\gamma}^{(2)}(u, \psi)$ being given by (3.14). We may take the limit $m=0$ in integral expression (4.1) to obtain the Green's function $D_{\gamma}^{(3)}\left(x, x_{0}\right)$,

$$
D_{\gamma}^{(3)}=\frac{1}{4 \pi^{2} B\left(2 \rho \rho_{0}\right)^{1 / 2}} \int_{\xi_{3}}^{\infty} \frac{1}{\left(\cosh u-\cosh \xi_{3}\right)^{1 / 2}} g_{\gamma}^{(2)}\left(u, \varphi-\varphi_{0}\right) d u .
$$

For the second step $n=4$, we first integrate by parts integral (4.1)

$$
\begin{aligned}
G_{\gamma}^{(3)}= & -\frac{1}{2 \pi^{2}\left(2 \rho \rho_{0}\right)^{1 / 2}} \int_{\xi_{3}}^{\infty} \frac{\sin \left[m\left(2 \rho \rho_{0}\right)^{1 / 2}\left(\cosh u-\cosh \xi_{3}\right)^{1 / 2}\right]}{m} \\
& \times g_{\gamma}^{(3)}\left(u, \varphi-\varphi_{0}\right) d u,
\end{aligned}
$$

where the function $g_{\gamma}^{(3)}(u, \psi)$ has the expression

$$
g_{\gamma}^{(3)}(u, \psi)=\frac{d}{d u}\left[\frac{1}{\sinh u} g_{\gamma}^{(2)}(u, \psi)\right] .
$$

With the aid of the Fourier cosine transform (The Fourier inverse is given in [15])

$$
\begin{aligned}
& \int_{-\infty}^{\infty} \frac{\sin \left[A\left(m^{2}+\lambda^{2}\right)^{1 / 2}\right]}{\left(m^{2}+\lambda^{2}\right)^{1 / 2}} \cos \left[\lambda\left(x^{2}-x_{0}^{2}\right)\right] d \lambda \\
& =\left\{\begin{array}{ll}
\pi J_{0}\left[m\left(A^{2}-\left(x^{2}-x_{0}^{2}\right)^{1 / 2}\right]\right. & A>x^{2}-x_{0}^{2} \\
0 & A<x^{2}-x_{0}^{2}
\end{array},\right.
\end{aligned}
$$

we perform the $\lambda$-integration in (2.5) and we obtain the integral expression

$$
\begin{aligned}
G_{\gamma}^{(4)}= & -\frac{1}{8 \pi^{2} B \rho \rho_{0}} \int_{\xi_{4}}^{\infty} J_{0}\left[m\left(2 \rho \rho_{0}\right)^{1 / 2}\left(\cosh u-\cosh \xi_{4}\right)^{1 / 2}\right] \\
& \times g_{\gamma}^{(3)}\left(u, \varphi-\varphi_{0}\right) d u,
\end{aligned}
$$

where the positive quantity $\xi_{4}$ is defined by

$$
\cosh \xi_{4}=\frac{\rho^{2}+\rho_{0}^{2}+\left(x^{1}-x_{0}^{1}\right)^{2}+\left(x^{2}-x_{0}^{2}\right)^{2}}{2 \rho \rho_{0}} .
$$


When the mass $m$ goes to zero, as $J_{0}(0)=1$, we obtain $D_{\gamma}^{(4)}\left(x, x_{0}\right)$ in terms of elementary functions as follows:

$$
D_{\gamma}^{(4)}=\frac{e^{i\left(\varphi-\varphi_{0}\right) \gamma} \sinh \left[\xi_{4}(1-\gamma) / B\right]+e^{-\imath\left(\varphi-\varphi_{0}\right)(1-\gamma)} \sinh \left[\xi_{4} \gamma / B\right]}{8 \pi^{2} B \rho \rho_{0} \sinh \xi_{4}\left[\cosh \left(\xi_{4} / B\right)-\cos \left(\varphi-\varphi_{0}\right)\right]}
$$

In four dimensions, we can compare with the results already known within the framework of the straight cosmic strings. For $\gamma=0$ and $\gamma=1 / 2$ (twisted field), integrals (4.6) reduce respectively to the ones of Linet $[12,17]$. In the case $B=1$ (Euclidean space), expression (4.8) of $D_{\gamma}^{(4)}\left(x, x_{0}\right)$ has been found by Bordag [18] for an arbitrary magnetic flux $\gamma$.

It is clear that we can continue to apply recurrence relation (2.5) to determine $G_{\gamma}^{(n)}\left(x, x_{0} ; m\right)$. We define the positive quantity $\xi_{n}$ by

$$
\cosh \xi_{n}=\frac{\rho^{2}+\rho_{0}^{2}+\left(x^{1}-x_{0}^{1}\right)^{2}+\cdots+\left(x^{n-2}-x_{0}^{n-2}\right)^{2}}{2 \rho \rho_{0}} .
$$

For even dimension, we have

$$
\begin{aligned}
G_{\gamma}^{(2 p)}= & \frac{1}{4 \pi B}\left[\frac{1}{\pi\left(2 \rho \rho_{0}\right)^{1 / 2}}\right]^{p-1}\left[-\frac{1}{\left(2 \rho \rho_{0}\right)^{1 / 2}}\right]^{p-1} \\
& \times \int_{\xi_{2 p}}^{\infty} J_{0}\left[m\left(2 \rho \rho_{0}\right)^{1 / 2}\left(\cosh u-\cosh \xi_{2 p}\right)^{1 / 2}\right] g_{\gamma}^{(2 p)}\left(u, \varphi-\varphi_{0}\right) d u
\end{aligned}
$$

where we have the recurrence relation $(p \geq 2)$

$$
g_{\gamma}^{(2 p)}(u, \psi)=\frac{d}{d u}\left[\frac{1}{\sinh u} g_{\gamma}^{(2 p-2)}(u, \psi)\right] \text {. }
$$

When $m=0$, it should be noted that all Green's functions $D_{\gamma}^{(2 p)}\left(x, x_{0}\right)$ for $p \geq 2$ can be expressed in closed form.

For odd dimensions, we have

$$
\begin{aligned}
G_{\gamma}^{(2 p+1)}= & \frac{1}{4 \pi B}\left[\frac{1}{\pi\left(2 \rho \rho_{0}\right)^{1 / 2}}\right]^{p}\left[-\frac{1}{\left(2 \rho \rho_{0}\right)^{1 / 2}}\right]^{p-1} \\
& \times \int_{\xi_{2 p+1}}^{\infty} \frac{\cos \left[m\left(2 \rho \rho_{0}\right)^{1 / 2}\left(\cosh u-\cosh \xi_{2 p+1}\right)^{1 / 2}\right]}{\left(\cosh u-\cosh \xi_{2 p+1}\right)^{1 / 2}} \\
& \times g_{\gamma}^{(2 p+1)}\left(u, \varphi-\varphi_{0}\right) d u
\end{aligned}
$$

where we have the relation

$$
g_{\gamma}^{(2 p+1)}(u, \psi)=g_{\gamma}^{(2 p)}(u, \psi)
$$




\section{Local Form of $G_{\gamma}^{(2)}\left(x, x_{0} ; m\right)$}

As mentioned in the introduction, the local form of $G_{\gamma}^{(2)}\left(x, x_{0} ; m\right)$ is defined for point $x$ close enough to point $x_{0}$. We work in the coordinate system $(\rho, \theta)$ in which the domain of validity of this local form will be

$$
\pi-2 \pi B<\theta-\theta_{0}<2 \pi B-\pi
$$

in the case $B>1 / 2$ in which we restrict ourselves. Restriction (5.1) is equivalent to (1.5).

We start from expression (3.9) of $\tilde{G}_{\gamma}^{(2)}$. We use another integral expression of the product of two Bessel functions [19]

$$
K_{i \nu}(m \rho) K_{\imath \nu}\left(m \rho_{0}\right)=\int_{0}^{\infty} K_{0}\left[m R_{2}(u)\right] \cos (\nu u) d u
$$

with

$$
R_{2}(u)=\sqrt{\rho^{2}+\rho_{0}^{2}+2 \rho \rho_{0} \cosh u}
$$

Hence, $\tilde{G}_{\gamma}^{(2)}$ can be rewritten under the form

$$
\tilde{G}_{\gamma}^{(2)}=\frac{1}{\pi^{2}} \int_{0}^{\infty} K_{0}\left[m R_{2}(u)\right] d u \int_{0}^{\infty} \sinh \pi \nu U_{\nu}(\theta) \cos \nu u d \nu
$$

The Fourier cosine transform appearing in (5.3) can be recast in the form

$$
\begin{aligned}
\int_{0}^{\infty} \sinh (\pi \nu) U_{\nu}(\theta) \cos (\nu u) d \nu \\
=\frac{1}{2} \int_{0}^{\infty} \frac{\cos (\nu u) d \nu}{\cosh (2 \pi \nu B)-\cos (2 \pi \gamma)} \\
\quad \times\left\{e^{2 i \pi \gamma} \cosh \left(\nu\left|\theta-\theta_{0}\right|+\pi \nu\right)-\cosh \left(\nu\left|\theta-\theta_{0}\right|-2 \pi \nu B+\pi \nu\right)\right. \\
\quad+e^{-2 i \pi \gamma} \cosh \left(\nu\left|\theta-\theta_{0}\right|-\pi \nu\right)-\cosh \left(\nu\left|\theta-\theta_{0}\right|+2 \pi \nu B-\pi \nu\right) \\
\left.\quad-2[\cos (2 \pi \gamma)-\cosh (2 \pi \nu B)] \cosh \left(\nu\left|\theta-\theta_{0}\right|-\pi \nu\right)\right\}
\end{aligned}
$$

by using the addition properties of the hyperbolic functions. The last line in (5.4) gives the usual Green's function since in this case $U_{\nu}(\theta)$ is given by (3.8). The other terms can be integrated with the aid of the formula [15]

$$
\begin{aligned}
& \int_{0}^{\infty} \frac{\cos (a \nu) \cosh (b \nu)}{\cosh (c \nu)+\cos d} d \nu \\
& \quad=\pi \frac{\cos [b(\pi-d) / c] \cosh [a(\pi+d) / c]-\cos [b(\pi+d / c] \cosh [a(\pi-d) / c]}{c \sin d[\cosh (2 \pi a / c)-\cos (2 \pi b / c)]}
\end{aligned}
$$


with the conditions of validity: $|b|<c, 0<d<\pi$ and $a>0$. Restriction (5.1) results from the first condition of validity. We suppose $\theta-\theta_{0}>0$. From the first line in (5.4), we get after some manipulations

$$
\frac{i}{2 B} \frac{e^{\imath\left(\theta-\theta_{0}+\pi\right) \gamma / B} \cosh [u(1-\gamma) / B]-e^{-i\left(\theta-\theta_{0}+\pi\right)(1-\gamma) / B} \cosh [u \gamma / B]}{\cosh (u / B)-\cos \left[\left(\theta-\theta_{0}+\pi\right) / B\right]} .
$$

From the second line in (5.4), we get after some manipulations

$$
-\frac{i}{2 B} \frac{e^{i\left(\theta-\theta_{0}-\pi\right) \gamma / B} \cosh [u(1-\gamma) / B]-e^{-\imath\left(\theta-\theta_{0}-\pi\right)(1-\gamma) / B} \cosh [u \gamma / B]}{\cosh (u / B)-\cos \left[\left(\theta-\theta_{0}-\pi\right) / B\right]} .
$$

The case $\theta-\theta_{0}<0$ is obtained from this result by putting $-\left(\theta-\theta_{0}\right)$ in the place of $\theta-\theta_{0}$ and by taking the complex conjugate.

We now turn to express $G_{\gamma}^{(2)}\left(x, x_{0} ; m\right)$ in the subset of the space defined by (1.5) for $B>1 / 2$. Combining the two previous expressions, we have thereby

$$
G_{\gamma}^{(2)}=\frac{1}{2 \pi} K_{0}\left(m r_{2}\right)+\frac{1}{4 \pi^{2} B} \int_{0}^{\infty} K_{0}\left[m R_{2}(u)\right] F_{B}^{(\gamma)}\left(u, \varphi-\varphi_{0}\right) d u
$$

where the function $F_{B}^{(\gamma)}(u, \psi)$ is

$$
\begin{aligned}
F_{B}^{(\gamma)}(u, \psi) & =i \frac{e^{i(\psi+\pi / B) \gamma} \cosh [u(1-\gamma) / B]-e^{-i(\psi+\pi / B)(1-\gamma)} \cosh [u \gamma / B]}{\cosh (u / B)-\cos (\psi+\pi / B)} \\
& -i \frac{e^{\imath(\psi-\pi / B) \gamma} \cosh [u(1-\gamma) / B]-e^{-\imath(\psi-\pi / B)(1-\gamma)} \cosh [u \gamma / B]}{\cosh (u / B)-\cos (\psi-\pi / B)}
\end{aligned}
$$

In the limit $\gamma=0, F_{B}^{(\gamma)}(u, \psi)$ given by (5.6) reduces to the the following function $F_{B}(u, \psi)$ already known from Garnir [16] and also Oberhettinger [20]

$$
F_{B}(u, \psi)=-\frac{\sin (\psi+\pi / B)}{\cosh (u / B)-\cos (\psi+\pi / B)}+\frac{\sin (\psi-\pi / B)}{\cosh (u / B)-\cos (\psi-\pi / B)} .
$$

In this case, we mention that the propagator (heat kernel) has been determined by Deser and Jackiw [21].

\section{Local Form of $G_{\gamma}^{(n)}\left(x, x_{0} ; m\right)$}

The application of recurrence relation (2.5) between the $G_{\gamma}^{(n)}\left(x, x_{0} ; m\right)$ is easy since the function $F_{B}^{(\gamma)}(u, \psi)$ does not depend on the mass $m$. We have only to know the usual Green's function in Euclidean space

$$
\frac{1}{(2 \pi)^{n / 2}} \frac{m^{n / 2-1}}{r_{n}^{n / 2-1}} K_{n / 2-1}\left(m r_{n}\right)
$$


where $r_{n}$ is the Euclidean distance between the two points $x$ and $x_{0}$. We find in the subset of the space defined by (1.5) for $B>1 / 2$,

$$
\begin{aligned}
G_{\gamma}^{(n)}= & \frac{1}{(2 \pi)^{n / 2}} \frac{m^{n / 2-1}}{r_{n}^{n / 2-1}} K_{n / 2-1}\left(m r_{n}\right) \\
& +\frac{m^{n / 2-1}}{(2 \pi)^{n / 2+1} B} \int_{0}^{\infty} \frac{K_{n / 2-1}\left[m R_{n}(u)\right]}{\left[R_{n}(u)\right]^{n / 2-1}} F_{B}^{(\gamma)}\left(u, \varphi-\varphi_{0}\right) d u
\end{aligned}
$$

with

$$
R_{n}(u)=\sqrt{\left(x^{1}-x_{0}^{1}\right)^{2}+\cdots+\left(x^{n-2}-x_{0}^{n-2}\right)^{2}+\rho^{2}+\rho_{0}^{2}+2 \rho \rho_{0} \cosh u} .
$$

In the massless cases for $n \geq 3$, we can take the limit where $m$ goes to zero. Taking into account the asymptotic behaviour of the $K_{\mu}$, we get

$$
\begin{aligned}
D_{\gamma}^{(n)}= & \frac{\Gamma(n / 2)}{2(n-2) \pi^{n / 2}} \frac{1}{r_{n}^{n-2}} \\
& +\frac{\Gamma(n / 2)}{4(n-2) \pi^{n / 2+1} B} \int_{0}^{\infty} \frac{1}{\left[R_{n}(u)\right]^{n-2}} F_{B}^{(\gamma)}\left(u, \varphi-\varphi_{0}\right) d u,
\end{aligned}
$$

where $\Gamma$ is the gamma function.

In 4-dimensional space, we can compare with the results already known within the framework of the straight cosmic strings. For $\gamma=0$ and $\gamma=1 / 2$ (twisted field), local forms (6.1) for $n=4$ reduce respectively to the ones of Linet $[12,17]$.

\section{Vacuum Energy-Momentum Tensor $(n=4)$}

Within the Euclidean quantum field theory of a complex scalar field in the spacetime of a straight cosmic string, the fundamental quantity for a massive scalar field is the Euclidean Green's function $G_{\gamma}^{(4)}\left(x, x_{0} ; m\right)$. We rewrite its expression (6.1) valid for $x$ close enough to point $x_{0}$ in the particular case $n=4$,

$$
G_{\gamma}^{(4)}\left(x, x_{0} ; m\right)=\frac{m K_{1}\left(m r_{4}\right)}{4 \pi^{2} r_{4}}+G_{\gamma}^{*(4)}\left(x, x_{0} ; m\right),
$$

where the regular term $G_{\gamma}^{*(4)}\left(x, x_{0} ; m\right)$ has the expression

$$
G_{\gamma}^{*(4)}=\frac{1}{8 \pi^{3} B} \int_{0}^{\infty} \frac{K_{1}\left[m R_{4}(u)\right]}{R_{4}(u)} F_{B}^{(\gamma)}\left(u, \varphi-\varphi_{0}\right) d u .
$$

The renormalization of expectation values of the energy-momentum tensor is performed by removing the usual Green's function in expression (7.1). Since the second term and its derivatives are regular in the coincidence limit $x=x_{0}$, we obtain simply for a locally flat spacetime

$$
\begin{aligned}
\left\langle T_{\mu}^{\nu}\right\rangle= & 2 \hbar c\left[(1-2 \xi) \nabla_{\mu} \nabla^{\nu_{0}}-2 \xi \nabla_{\mu} \nabla^{\nu}\right. \\
& \left.+\left(2 \xi-\frac{1}{2}\right) \delta_{\mu}^{\nu}\left(m^{2}+\nabla_{\alpha} \nabla^{\alpha_{0}}\right)\right]\left.G_{\gamma}^{*(4)}\right|_{x=x_{0}},
\end{aligned}
$$


$\xi$ being the coupling parameter, and $\nabla_{\mu}\left(\nabla_{\mu_{0}}\right)$ denotes the covariant differentiation with respect to the coordinate $x^{\mu}\left(x^{\mu_{0}}\right)$.

In the massive case, the asymptotic form of the vacuum energy-momentum for large $\rho$ can be evaluated. The calculations are simplified by remarking that the derivatives $\left.\nabla_{\varphi} \nabla^{\varphi} G_{\gamma}^{*(4)}\right|_{x=x_{0}}$ and $\left.\nabla_{\varphi} \nabla^{\varphi_{0}} G_{\gamma}^{*(4)}\right|_{x=x_{0}}$ can be determined from identity $\left(\nabla_{\alpha} \nabla^{\alpha}-m^{2}\right) G_{\gamma}^{*(4)}=0$. By keeping only the coefficients containing $1 / \rho$ of the Bessel functions in the expression of the component $\left\langle T_{\tau}^{\tau}\right\rangle$, we find

$$
\left\langle T_{\tau}^{\tau}\right\rangle \simeq(4 \xi-1) \frac{m^{3}}{16 \pi^{3} B} \int_{0}^{\infty} \frac{3 K_{1}+K_{3}}{\rho}(1+\cosh u)^{\frac{1}{2}} F_{B}^{(\gamma)}(u, 0) d u .
$$

Taking into account the asymptotic form of $K_{1}$ and $K_{3}$, we find

$$
\left\langle T_{\tau}^{\tau}\right\rangle \sim(4 \xi-1) \frac{m^{3} \sqrt{\pi}}{4 \pi^{3} B \sqrt{2 \rho}} \int_{0}^{\infty} e^{-\sqrt{2} m \rho(1+\cosh u)^{\frac{1}{2}}}(1+\cosh u)^{\frac{1}{2}} F_{B}^{(\gamma)}(u, 0) d u
$$

for $m \rho \gg 1$. The limit of the integral is performed by using Laplace's method, we obtain

$$
\left\langle T_{\tau}^{\tau}\right\rangle \sim(1-4 \xi) \frac{m^{2}}{8 \pi^{2} B} \frac{\sin [\pi \gamma / B]+\sin [\pi(1-\gamma) / B]}{1-\cos (\pi / B)} \frac{\exp (-2 m \rho)}{\rho^{2}} .
$$

Since spacetime (1.1) is globally static, we have $\left\langle T_{\tau}^{\tau}\right\rangle=\left\langle T_{t}^{t}\right\rangle$ since $t=-i \tau$.

The asymptotic form of the other components for large $\rho$ can be also evaluated. We have finally

$$
\left\langle T_{\mu}^{\nu}\right\rangle \sim\left\langle T_{t}^{t}\right\rangle \operatorname{diag}(1,1,0,1) \text { for } m \rho \gg 1
$$

For $B=1$, Serebriany [4] has found the same form (7.5), likewise Shiraishi and Hirenzaki [23] for $B \neq 1$ but in the case $\gamma=0$ and $\xi=1 / 6$.

In the massless case, we rewrite expression (6.3) in the particular case $n=4$ under the form

$$
D_{\gamma}^{(4)}\left(x, x_{0}\right)=\frac{1}{4 \pi^{2} r_{4}^{2}}+D_{\gamma}^{*(4)}\left(x, x_{0}\right)
$$

where the regular term $D_{\gamma}^{*(4)}\left(x, x_{0}\right)$ has the expression

$$
D^{*(4)}=\frac{1}{8 \pi^{3} B} \int_{0}^{\infty} \frac{1}{\left[R_{4}(u)\right]^{2}} F_{B}^{(\gamma)}\left(u, \varphi-\varphi_{0}\right) d u .
$$

By inserting (7.7) into formula (7.3) with $m=0$, we can express $\left\langle T_{\mu}^{\nu}\right\rangle$ in the coordinate system $\left(x^{1}, x^{2}, \rho, \varphi\right)$ under the form

$$
\begin{aligned}
\left\langle T_{\mu}^{\nu}\right\rangle= & {\left[w_{4}(\gamma)-\frac{1}{3} w_{2}(\gamma)\right] \frac{\hbar c}{\rho^{4}} \operatorname{diag}(1,1,1,-3) } \\
& +4\left(\xi-\frac{1}{6}\right) w_{2}(\gamma) \frac{\hbar c}{\rho^{4}} \operatorname{diag}\left(1,1,-\frac{1}{2}, \frac{3}{2}\right)
\end{aligned}
$$


where the derivative $\left.\nabla_{\varphi} \nabla^{\varphi} D_{\gamma}^{*(4)}\right|_{x=x_{0}}$ and $\left.\nabla_{\varphi} \nabla^{\varphi_{0}} D_{\gamma}^{*(4)}\right|_{x=x_{0}}$ having been calculated from the identity $\nabla_{\alpha} \nabla^{\alpha} D_{\gamma}^{*(4)}=0$. The quantities $w_{2}(\gamma)$ and $w_{4}(\gamma)$ are the following integrals

$$
\begin{aligned}
& w_{2}(\gamma)=\frac{1}{8 \pi^{3} B} \int_{0}^{\infty} \frac{1}{(1+\cosh u)} F_{B}^{(\gamma)}(u, 0) d u \\
& w_{4}(\gamma)=\frac{1}{8 \pi^{3} B} \int_{0}^{\infty} \frac{1}{(1+\cosh u)^{2}} F_{B}^{(\gamma)}(u, 0) d u
\end{aligned}
$$

where $F_{B}^{(\gamma)}(u, 0)$ appearing in (7.9) and (7.10) is deduced from (5.6),

$$
F_{B}^{(\gamma)}(u, 0)=-2 \frac{\sin [\pi \gamma / B] \cosh [u(1-\gamma) / B]+\sin [\pi(1-\gamma) / B] \cosh [u \gamma / B]}{\cosh (u / B)-\cos (\pi / B)} .
$$

Integrals of type (7.9) or (7.10) have been studied by Dowker [8, 22]. We get thus the explicit expressions

$$
\begin{aligned}
w_{2}(\gamma)= & -\frac{1}{8 \pi^{2}}\left\{\frac{1}{3}-\frac{1}{2 B^{2}}\left[4\left(\gamma-\frac{1}{2}\right)^{2}-\frac{1}{3}\right]\right\} \\
w_{4}(\gamma)= & -\frac{1}{720 \pi^{2}}\left\{11-\frac{15}{B^{2}}\left[4\left(\gamma-\frac{1}{2}\right)^{2}-\frac{1}{3}\right]\right. \\
& \left.+\frac{15}{8 B^{4}}\left[16\left(\gamma-\frac{1}{2}\right)^{4}-8\left(\gamma-\frac{1}{2}\right)^{2}+\frac{7}{15}\right]\right\}
\end{aligned}
$$

which are only valid for $B>1 / 2$. Incidentally, the coefficient in front of $1 / B^{4}$ in form (7.12) has been corrected. We can then verify that vacuum energy-momentum tensor (7.8) with quantities (7.11) and (7.12) agrees with the result given by Frolov and Serebriany [24]. For $\gamma=0$, we find again the known result [10-12]. For massless twisted scalar field $\left(\gamma=\frac{1}{2}\right)$, expression (7.8) reduces to the one of Smith [11].

\section{Conclusion}

In metric (1.1), we have determined the Green's function $G_{\gamma}^{(n)}\left(x, x_{0} ; m\right)$ for a massive scalar field subject to requirements (1.3), characterized by the fractional part $\gamma$ of the magnetic flux around the line singularity. We have given integral expression (4.10) in even dimension and (4.12) in odd dimension for $G_{\gamma}^{(n)}\left(x, x_{0} ; m\right)$. In the massless case, the Green's function $D_{\gamma}^{(2 p)}\left(x, x_{0}\right)$ for $p>1$ can be expressed in terms of elementary functions. For point $x$ close enough to point $x_{0}$, we have obtained a local form (5.5) for the Green's function $G_{\gamma}^{(n)}\left(x, x_{0} ; m\right)$. It should be emphasized that this local form is convenient, for instance, to evaluate the expectation values of the energy-momentum tensor in quantum field theory. As an application we have performed explicitly this calculation for a massless field. We calculate only with finite quantities which are definite integrals and therefore we might perform a numerical analysis in the case of a massive scalar field. We have presently derived the asymptotic form of this tensor at large distance. It is interesting to point out that this procedure can be applied systematically to all dimensions. For exemple, in the three dimensional case, when $\gamma=0$ and in the limit where $B \rightarrow 1$, we can easily see that we obtain again the results of Guimarães and Linet [25] for a massive scalar field. 


\section{References}

1. Staruszkiewicz, A.: Acta Phys. Polon. 24, 735 (1963)

2. Gott III, J.R.: Astrophys. J. 288, 422 (1985) Hiscock, W.A.: Phys. Rev. D 31, 3288 (1985)

Linet, B.: Gen. Rel. Grav. 17, 1109 (1985)

3. Goldin, G.A. Menikoff, R., Sharp, D.H.: J. Math. Phys. 22, 1664 (1981) Wilczek, F.: Phys. Rev. Lett. 48, 1144 (1982)

4. Serebriany, E.M.: Theor. Math. Phys. 64, 846 (1985)

5. De Sousa Gerbert, P., Jackiw, R.: Commun. Math. Phys. 124, 229 (1989) Kay, B.S., Studer, U.M.: Commun. Math. Phys. 139, 103 (1991)

6. De Sousa Gerbert, P.: Phys. Rev. D 40, 1346 (1989) Coutinho, F.A.B., Fernando Perez, J.: Phys. Rev. D 48, 932 (1993)

7. Dowker, J.S.: J. Phys. A 10, 115 (1977)

8. Dowker, J.S.: Phys. Rev. D 36, 3095 (1987)

9. Vilenkin, A.: Three hundred years of gravitation. S.W. Hawking, W. Israel (eds.). Cambridge: Cambridge University Press 1987, p. 499

10. Helliwell, T.M., Konkowski, D.A.: Phys. Rev. D 34, 1918 (1986)

11. Smith, A.G.: The formation and evolution of cosmic strings. G.W. Gibbons, S.W. Hawking, T. Vaschapati (eds.). Cambridge: Cambridge University Press 1990, p. 262

12. Linet, B.: Phys. Rev. D 35, 536 (1987)

13. DeWitt, B.S.: Relativity, groups and topology II. B.S. DeWitt, R. Stora (eds.). Amsterdam: North-Holland 1984, p. 381

14. Gibbons, G.W.: General relativity: an Einstein centenary survey. S.W. Hawking, W. Israel (eds.). Cambridge: Cambrige University Press 1979, p. 639

15. Gradshteyn, I.S., Ryzhik, I.M.: Tables of integrals, series, and products. New York: Academic Press 1980

16. Garnir, H.G.: Bull. Soc. R. Sci. Liège 21, 119 and 207 (1952)

17. Linet, B.: Int. J. Mod. Phys. D 1, 371 (1992)

18. Bordag, M.: Ann. Phys. (N-Y) 206, 257 (1991)

19. Erdélyi, A.: Higher transcendental functions. Vol. 2. Malabar: Krieger 1981

20. Oberhettinger, F.: Comm. Pure Appl. Math. 7, 551 (1954)

21. Deser, S., Jackiw, R.: Commun. Math. Phys. 118, 495 (1988)

22. Dowker, J.S.: Phys. Rev. D 36, 3742 (1987)

23. Shiraishi, K., Hirenzaki, S.: Class. Quantum Grav. 9, 2277 (1992)

24. Frolov, V.P., Serebriany, E.M.: Phys. Rev. D 35, 3779 (1987)

25. Guimarães, M.E.X., Linet, B.: Class. Quantum Grav. 10, 1665 (1993)

Communicated by G. Felder 